\title{
Polymeric Micelles: Morphology, Synthesis, and Pharmaceutical Application
}

\author{
Kun $\mathrm{Bai}^{1, \dagger}$, Anda Wang ${ }^{2, *}, \dagger$ \\ ${ }^{1}$ Queen Mary University of London Engineering School, Northwestern Polytechnical University, Xi'an, Shaanxi Province, China. \\ 21010 West University Ave, Urbana, IL, 61801, USA. \\ ${ }^{\dagger}$ These authors contributed equally.
}

\begin{abstract}
Polymeric micelles (PMs) are nanosized core-shell molecules formed by the self-assembly of amphiphilic block copolymers. Their unique morphologies, biocompatibility have allowed them to serve as drug carriers and have found wide applications in the pharmaceutical industry. This review summarizes the recent progress of PM development and its applications in the pharmaceutical field. Firstly, we discussed the fundamentals of polymeric micelles, the formation mechanism, and their relative morphologies. Then we listed various common synthetic methods, including the solvent-free method, solvent-switch method, microfluid method, etc. Secondly, we illustrated the application of polymeric micelles in pharmaceutical applications on targeting, imaging, and stimuli-responsive drug release. In the end, we summarize the fundamental aspect of how to prepare polymeric micelles, their resulting morphology, and their applications in the medical field. Finally, we provide insights into polymeric micelles' future developments on their longer shelf life and better environmental adaptability.
\end{abstract}

\section{Introduction}

Polymeric micelles (PMs) are nanosized polymer capsules with membranes generally considered to have a hydrophobic bilayer structure similar to phospholipids [1] In the last two decades, PMs have experienced rapid development [2-8]. Various polymeric micelles have been synthesized, and they have found potential applications in target positioning and as drug carriers for cancer, gene therapy, and even catalysts and building materials.

In this review, we summarized the recent progress of synthesis and applications of these PMs. We first discuss the basic definition of polymeric micelles, followed by their formation mechanism and relative morphology. Testing methods of CMC (critical micelles concentration) were introduced, which significantly impacted the synthesis of polymeric micelles. The morphology of PMs was determined by several factors, including the ratio of hydrophobic to hydrophilic segments, temperature, and concentration of the solution.

We further introduced synthetic methods, which are an important subject in the study of polymer vesicles. There are many alternative methods to prepare PMs. Among them, solvent-switching techniques and solventfree techniques are the most widely used technologies [5], but they still have many drawbacks. Therefore, this review will also introduce some other new technologies in recent years, including PISA (Polymerization-induced self-assembly) [3-6]. In addition, complex or large vesicles are also a hot topic in recent years. The synthesis mechanism and technology of some such vesicles will also be introduced $[7,8]$.

Finally, several pharmaceutical applications of PMs would be discussed in this review. The most important contribution is its applications as drug carriers due to its high similarity to cell membranes. However, after the drug enters the blood vessel, the cells that need to be released must be accurately located. Otherwise, serious side effects may occur. Therefore, some tumor-targeting technologies and applications, including passive targeting and active targeting, will be introduced in this article. Additionally, PM imaging methods include nuclear imaging, CT, and MRI, which are also briefly mentioned. Finally, the review will discuss several stimulusresponsive PMs used as drug carriers in detail.

\section{Fundamentals of PMs}

\subsection{Micelles}

According to IUPAC, micelles are "particles of colloidal dimensions that exist in equilibrium with the molecules or ions in solutions from which it is formed." Micelles have great application value in the pharmaceutical field because they can carry drugs into the liquid to make some slightly soluble or insoluble substances more soluble. The hydrophilic head is generally combined with the solution

*Corresponding author: anda2@illinois.edu 
and self-assembled into a spherical shape in the aqueous solution. Aggregation number is an index used to describe the average number of monomers required to form a micelle, usually between 50-200. The formation of micelles is determined by the number and morphology of amphiphilic molecules and the temperature and properties of the solvent. Continuously adding amphiphilic molecules to the solvent, when the concentration of amphiphilic molecules exceeds a certain value, they will self-assemble to form micelles. The driving force for the formation of micelles is the difference in the free energy of the system. This specific concentration is called the critical micelle concentration (CMC). Below CMC, amphiphilic molecules will preferentially gather at the airwater interface. At $\mathrm{CMC}$, the amphiphilic molecules adsorbed at the air-water interface are saturated, and micelle monomers begin to form [9].

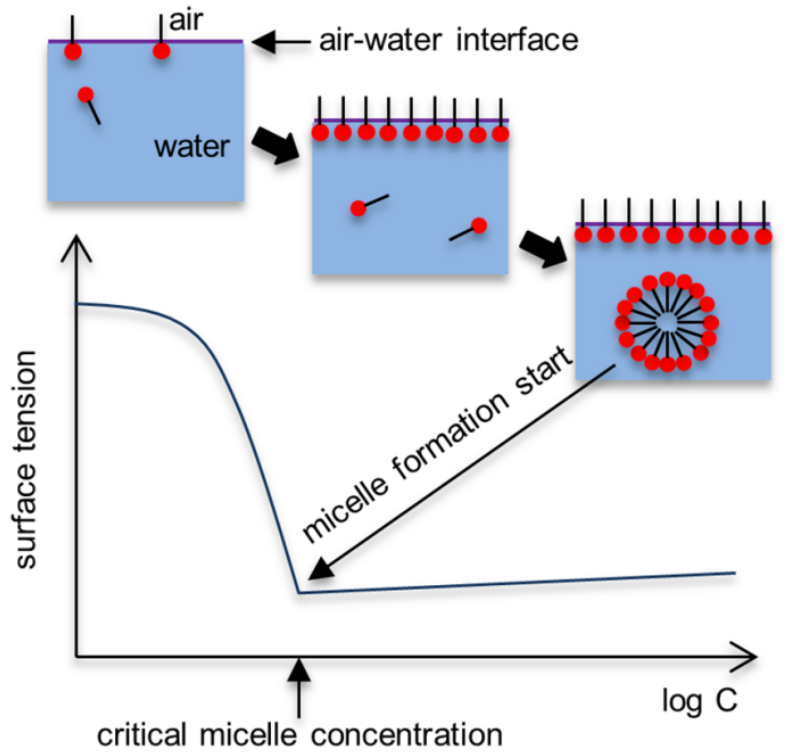

Fig. 1. Surface tension and surfactant concentration relationship. After CMC, the surface tension no longer changes, which indicates that the amphiphilic molecules on the surface of the solution are saturated [10].

\subsection{Polymeric micelles}

Polymeric micelles are a type of micelles, but the amphiphilic molecules formed in solution are macromolecules. According to the definition of IUPAC, polymer micelles are "organized self-assembled bodies formed in liquid and composed of amphiphilic macromolecules, usually amphiphilic and amphiphilic blocks made of hydrophilic and hydrophobic blocks, diblock or triblock copolymer." PMs are usually spherical. Compared with traditional micelles, PMs have the following characteristics. (1) Larger volume, usually 10$100 \mathrm{~nm}$ in diameter. (2) Lower CMC, but still in equilibrium with isolated macromolecules. (3) PMs show better thermodynamic and kinetic stability. [11] In addition, due to the biological stability and diversity of polymers, the hydrophilic and hydrophobic blocks of PMs are usually easier to design. For instance, the hydrophilic shells originate from poly (ethylene glycol) (PEG), poly(vinyl alcohol) (PVA), poly(N-vinyl-2-pyrrolidone) (PVP), poly(-acrylic acid), poly(acrylamide) (PAAm), poly(glycerol) (PG), poly(amino acid) (PAA), and polysaccharides. The hydrophobic blocks can usually be designed by polyesters (i.e., poly (glycolic acid) (PGA), poly(ecaprolactone) (PCL), and poly (D, L-lactic acid) (PDLLA)) or polyethers (i.e., poly (ethylene oxide) (PEO) and poly(propylene oxide) (PPO)). [12]

\subsection{Formation mechanism}

Similar to the formation mechanism of micelles, the formation of PMs is also based on the self-assembly of amphiphilic compounds. The resulting micelles consist of a hydrophilic block shell and a hydrophobic block core. After reaching the $\mathrm{CMC}$, PMs can also self-assemble generation. Compared with general micelles, the advantage of PMs is that their hydrophobic blocks are larger and consequently have a lower CMC value. Therefore PMs are more stable in dilute solutions. During the formation process below the CMC, the hydrophobic block will avoid contact with the solution. Because of that, the amphiphilic macromolecules will be concentrated at the air-solution interface. When CMC is reached, the hydrophobic heads of amphiphilic macromolecules will gather into nuclei due to the saturation of the air-solution interface. This can be explained by the "like dissolve like" theory. In addition, to keep the hydrophilic block in contact with the solution at all times, it will generate an inward force, ensuring that the amphiphilic macromolecules will not combine indefinitely into a single phase. Therefore, PM is formed by the balance of two opposite forces. Besides CMC, there is another important indicator called the critical micelle temperature (CMT). Only when the temperature is greater than this critical temperature, and the concentration is greater than $\mathrm{CMC}$, polymeric micelles can self-assemble and form.
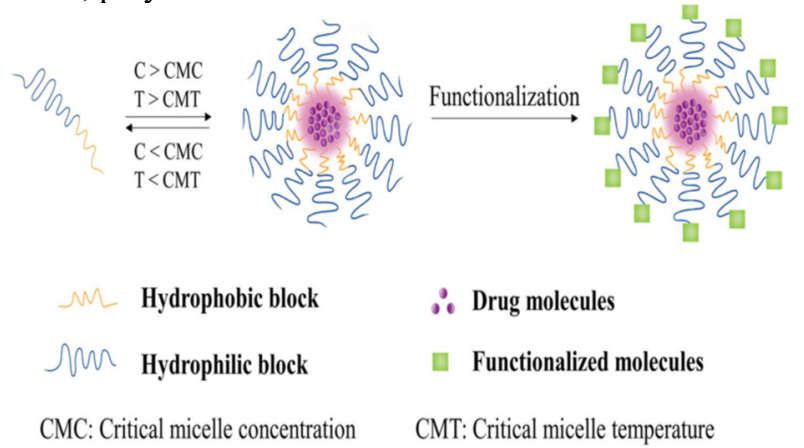

Fig. 2. Mechanism of polymeric micelles formation [12].

\subsection{Morphology}

\subsubsection{Typical morphology of polymeric micelles}

The shape of polymeric cells is mostly determined by the size ratio of the hydrophobic segment to the hydrophilic segment and the concentration of surfactants. When block copolymer in dilute solution, they will likely form the morphology of spherical shape [13]. In most block copolymers, the hydrophilic group will have a larger size than the hydrophobic group. This feature results in a large hydrophilic "head group" and forms a cone shape as a hydrophobic group is located at the cone's tip. All the 
hydrophobic tips gathered to form a spherical shape. Shapes of micelle other than spherical (rods, vesicles, etc.) can be produced by increasing temperature or concentration [14].

\subsubsection{Morphology of polymeric micelles in drug- delivery application}

The effect of different shapes of polymeric micelles on drug delivery remained mainly unexplored. Spheric polymeric micelles are the most explored ones for drug delivery [15]. However, polymeric micelles other than spherical shape in drug delivery are also worth further research. Since polymeric micelles are required to release drugs under specific conditions and specific time lengths, the morphology of polymeric micelles can be used as one of the physical parameters to improve their effectivity. For example, rod shapes have different loading capacities compare to spherical shapes. Moreover, rod shapes are more suitable in the aerosol formulation in the lung compare to spherical shapes because their slim tubular structures are more easily expand in the lung

\section{Synthesis}

To prepare polymersomes, six synthesis methods could be used. The most commonly used method is Solvent-switch technique and organic solvent-free technique. The former needs to dissolve polymers into the organic solvent, while the latter only needs to dissolve polymers into the water. This paper will also introduce other preparation methods such as polymerization-induced self-assembly (PISA), centrifugation-induced self-assembly, and the microfluid method.

\subsection{Solvent-switch methods}

The solvent-switch method is also known as the "phase inversion" technique. Using this method, firstly, polar organic solvents such as N,N-dimethylformamide (DMF), and tetrahydrofuran (THF) are needed for the solution of polymers $[1,2,17,18]$. Then slowly add the second solvent (usually water) to the polymer solution or inject the polymer solution into the second solvent to hydrate the solution. Due to the insolubilities of the hydrophobic segment of the amphiphilic copolymer water, it will increase the interfacial tension between the hydrophobic segment and water, thereby making the copolymer selfassemble to form polymer vesicles. To change the particle size and distribution of polymer vesicles, different solvents can be chosen. However, the polymersomes prepared by this method is usually contained considerable residual organic solvent, which required further purification. This step is highly time-consuming and will increase the cost. In addition, as mentioned above, although changing the solvent can change the particle size and distribution of polymer vesicles, the change like the solvent during the dialysis process can also cause unexpected morphological changes.

\subsection{Solvent-free methods}

\subsubsection{Rehydration method}

The rehydration method [19] usually contains film swelling and bulk swelling, sonication, and extrusion. For film swelling, the first step is similar to the solvent-switch method. Polymers need to dissolve into an organic solvent such as chloroform, followed by the evaporation of organic solvent to form a thin film. During the hydration, the water is used to penetrate the polymer di-layers by the hydration interaction force made the copolymer swell and extrude, and self-assemble to form polymer vesicles with larger particle size and wider distribution. Some specific methods can be used to improve the properties of polymer vesicles. Generally, the polymer vesicles are squeezed through filters of different pore sizes under high pressure to produce the vesicles with smaller particle size and distribution [20]. Additionally, Battaglia and Ryant increase the speed of water diffusion into the polymer film through an alternating electric field action, thereby preparing polymer vesicles with a narrow particle size distribution [21].

For bulk swelling, with the assistance of some watersoluble polymers, there is no need to use organic solvents when preparing polymer vesicles.[22] Jianzhong and Steven P. Armes reported a method for preparing block copolymer vesicles in pure water by preparing and using poly(e-caprolactone)-block-poly[2-(methacryloyloxy) ethyl phosphorylcholine], PCL-b-PMPC, and then through the process of sol-gel chemical stabilization in the capsule membrane [23].

Ani T. Nikova et al. also reported a method of "stuffing" the aliphatic region of self-assembled aggregates with hydrophobic homopolymers to more easily prepare self-assembled macromolecular structures with controllable properties. The filled membranes made of hydrates and hydrophobic homopolymers are hydrated, and "filled" vesicles and various emulsions can be formed. The film thickness of micelles can be adjusted by changing the content of hydrophobic homopolymer in the polymer mixture [23].

\subsection{2 $\mathrm{pH}$ tuning in pure water}

For $\mathrm{pH}$-sensitive polymer, polymersomes can be formed by increase or decrease the $\mathrm{pH}$ value of solution due to the ratio of hydrophobic section and hydrophilic section will change with $\mathrm{pH}$ value, and polymer could self-assemble polymersomes. For instance, Jianzhong Du et al. reported a method for successfully preparing polymer vesicles using A Biocompatible Zwitterionic Copolymer, PMPC25-b-PDPA120 diblock copolymer. By increasing the $\mathrm{pH}$ of the solution, the self-assembly formation of polymer vesicles can be observed in TEM [24,25]. 

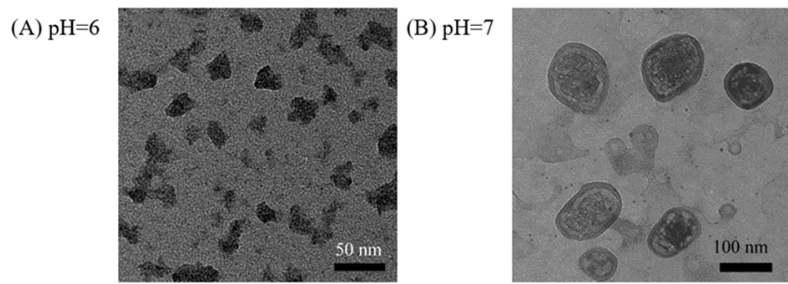

(C) $\mathrm{pH}=8.3$

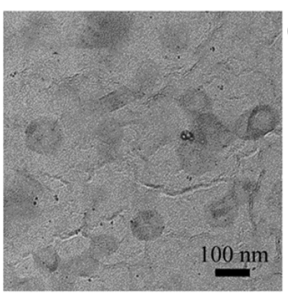

(D) $\mathrm{pH}=10$

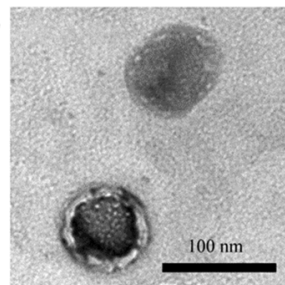

Fig. 3. TEM images obtained using PMPC25-b-PDPA120 diblock copolymer at different solution $\mathrm{pH}$. (A) At $\mathrm{pH}=6.0$, no obvious vesicles are observed in the microstructure. (B) At $\mathrm{pH}=7.0$, a few small vesicles appear. (C) At $\mathrm{pH}=8.3$, more vesicles appear. (D) At $\mathrm{pH}=9.0$, a completely large vesicle structure appears.

\subsubsection{PICsomes}

PICsomes (Polyion Complex Vesicles), first developed by Kishimura et al. [26-30,33-35]. It is formed by the selfassembly of a complex of oppositely charged polyelectrolytes in an aqueous solution. PICsomes are simple to prepare, have semi-permeability and high biocompatibility, and provide technical support for the future use of polymer vesicles as drug carriers. Akihiro Kishimura introduced a method for preparing nanoPICsomes. Compared with traditional PICsomes, nanoPICsomes have a uniform structure and can control their size. Using homopolymer with fPEG value $\leqslant 10 \%$ to form PIC, mixing homo-P (Asp-AP) and PEG-b-PAsp ( $f P E G \sim 8 \%$ ), the desired hollow vesicles and vesicle wall structure was obtained. It is a PEG-PIC-PEG three-layer structure with a size range of up to $300 \mathrm{~nm}$. This achievement is crucial for future applications in nanomedicine [3]. Takahashi et al. also reported that by adding anionic neutral di-hydrophilic block copolymer (AP) and cationic neutral di-hydrophilic block copolymer (MP) to the solution to achieve classically unstable reversibility, vesicles and spherical gels could be produced. The morphology between the bundle's changes and forms PICsomes.[30]

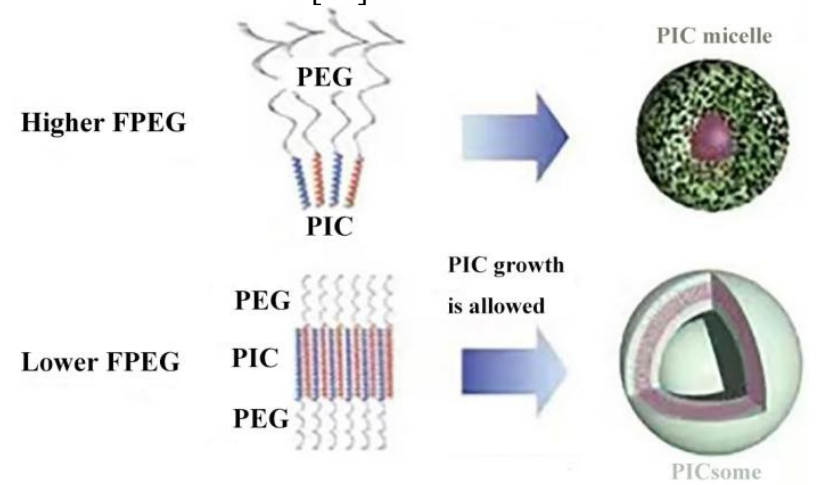

Fig. 4. Schematic representations of the structural control of polyion complex (PIC) assemblies.[3]

\subsection{Other synthesis methods}

\subsubsection{Polymerization-induced self-assembly (PISA):}

General polymer self-assembly needs to be carried out in a dilute solution with a volume fraction of less than $1 \%$, reducing the yield and limiting its use in practical applications [3-6]. Efficient synthesis of block copolymer vesicles with fixed morphology in concentrated solution is recognized as a solvable technical problem. Recently, amphiphilic diblock copolymers can be formed by radical polymerization of water-immiscible monomers (such as styrene, methyl methacrylate, or n-butyl acrylate) [31]. The general PISA reaction mechanism is shown in Fig. 5.

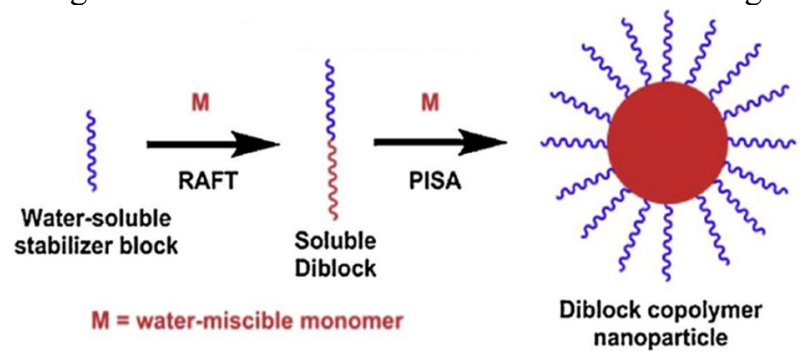

Fig. 5. Mechanism and example of polymerization-induced self-assembly in aqueous solution [6].

For example, A Blanazs reported a method of selfassembled vesicles using poly(glycerol monomethacrylate)-blockpoly(2-

hydroxypropylmethacrylate), PGMA-b-PHPMA, through reversible addition-fragmentation chain transfer (RAFT). In this case, self-assembly and polymerization reactions occur simultaneously. Since the PGMA block is hydrophobic, as the polymerization reaction progresses, there are more and more PHPMA blocks, making the ratio of hydrophilic and hydrophobic ends larger and larger, and promotes the progress of self-assembly [4]. However, only hydrophilic monomers that form a hydrophobic polymer after the polymerization reaction can use this method.

PISA is an important method to form polymer vesicles, which solves the low efficiency of traditional methods. It can be self-assembled in a concentrated solution. It has great application potential for high-efficiency microencapsulation carriers and as drug carriers [36].

\subsubsection{Centrifugation-induced self-assembly}

Centrifugation is an important way to form vesicles. In the simplest case, applying agitation or centrifugation in a water/oil or oil/water system can produce complex vesicles. Inspired by this, stable polymer vesosomes (polymersomes in polymersomes) can be prepared by simple centrifugation. Using oil phase dispersion PB-bPEO diblock copolymer and water phase as aqueous sucrose $(380 \mathrm{mOsm})$ solution to produce $\mathrm{W} / \mathrm{O}$ emulsion followed by pouring it into another water phase as aqueous sucrose $(380 \mathrm{mOsm})$ solution. With centrifugation and gravity force assistance, $\mathrm{W} / \mathrm{O}$ droplets would come out and penetrate the emulsion to form polymer vesosome macromolecules [7]. 


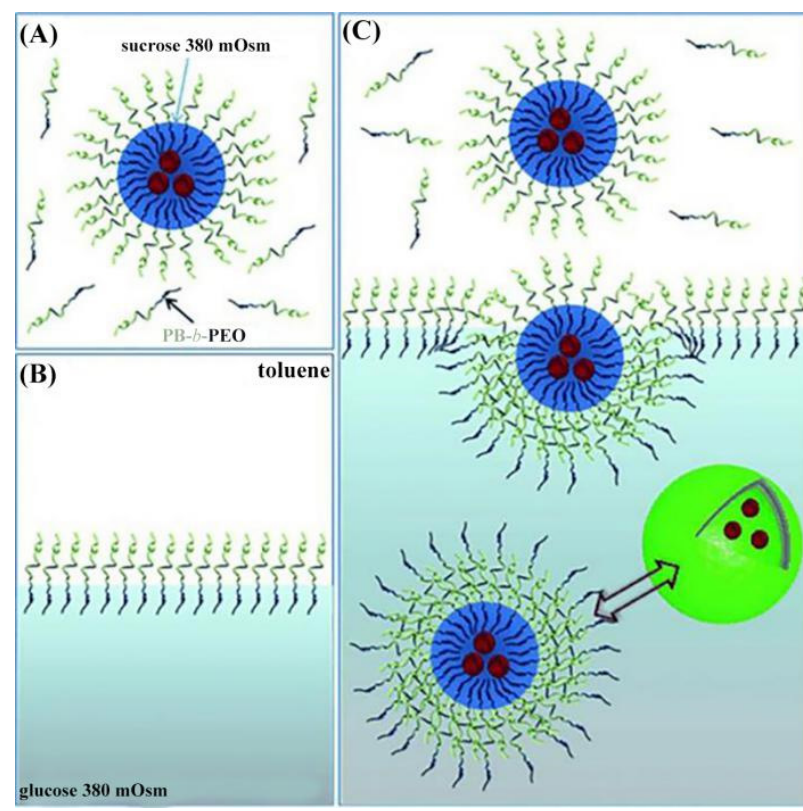

Fig. 6. Schematic representation of the centrifugation-induced process generating giant polymersomes or polymer vesosomes [8].

\subsubsection{Microfluid method}

The Microfluid method is another significant method to prepare giant polymer vesosomes. The mechanism of producing macromolecular vesicles is similar to that of centrifugation [32]. Both force the water-in-oil system to pass through the oil-in-water system to form a $\mathrm{W} / \mathrm{O} / \mathrm{W}$ system or a more complex system. This method uses a typical device, as shown in Fig. 7. The left arm is an O/W emulsion. The ring structure allows the water-in-oil system to pass through the aqueous solution and form stable droplets. At the "three forks", the water-in-oil colloid containing the droplets and the aqueous solution meet and form a stable fluid. After the fluid is stabilized, the triangular column guides the water at the position indicated by the green circle to make the aqueous droplets enter the water from the oil phase and form large vesicles [31].

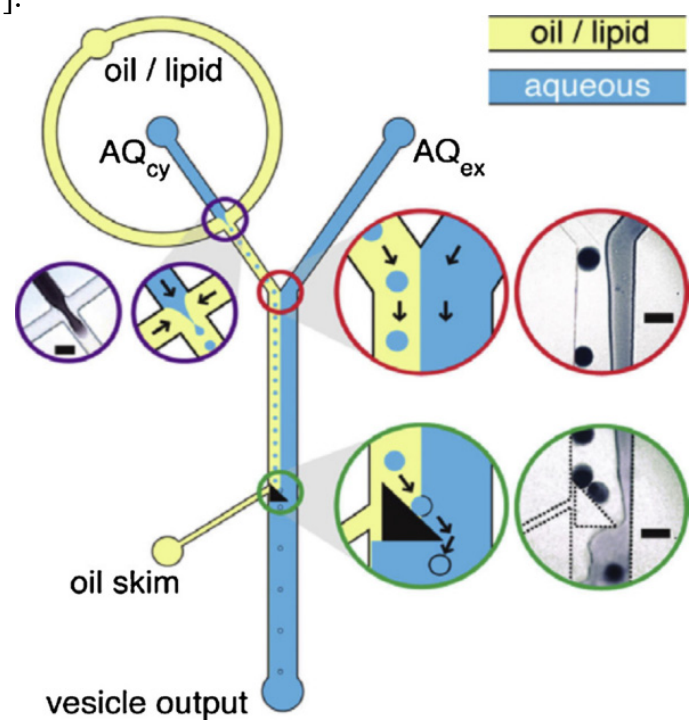

Fig. 7. Microfluid device schematic diagram

\section{Pharmaceutical application}

Nowadays, around 90 percent of drugs generated by the pharmaceutical industry have poor water solubility [37]. While anti-cancer drug potency will be greatly reduced after oral administration, intravenous administration of these drugs is also challenging due to the specific formulation of organic solvent and surfactants [38]. This challenge result in the failure of reaching the market due to their poor solubility even though they have desired pharmaceutical properties. Recent research of polymeric micelles, however, provides an alternate novel way to deliver these medicines. Drugs can be loaded by trap the target in the hydrophobic core of polymeric micelles.

\subsection{Tumor targeting}

Polymeric micelles are generally more stable in physiological solutions than surfactant micelles because Polymeric micelles are formed from amphiphilic block copolymers [37]. The small size $(<100 \mathrm{~nm})$ of polymeric micelle gives a surface-smoothing effect and allows longer retention time circulating in the blood and results from a higher accumulation of drug at the target site. Passive and active targeting has been used as delivery strategies to target anti-cancer drugs to interested physiological sites. Those strategies can improve their therapeutic index and reduce the harm to other non-target organs.

(A) Non-targeted micelle (Passive targeting)

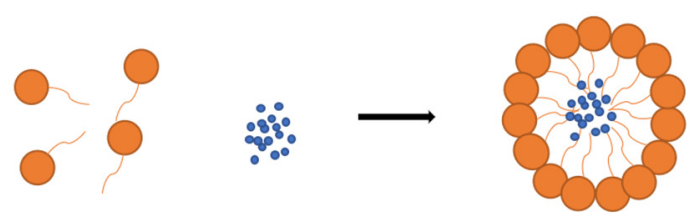

(B) Targeted micelle (Acitive targeting)

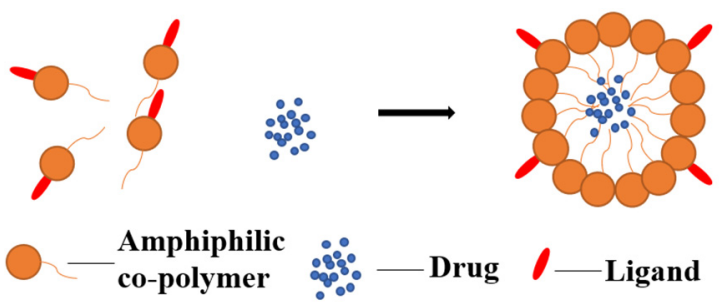

Fig. 8. Assemble scheme for (A) non-targeted PM used for passive targeting and (B) targeted PM used for active targeting

\subsubsection{Passive targeting}

It is now a well-known fact that under certain conditions such as inflammation or hypoxia, which is typical for tumors, the endothelium of blood vessels becomes more permeable compared to normal blood vessels [39]. Those blood vessels in tumor sites will leakier than normal ones due to their incomplete tumor vasculature culture. Therefore, those cultures allow selectively enhanced permeation of macromolecules, but small molecule drugs will not be affected due to their short circulation time. This phenomenon is called the EPR effect. Thus, 
polymeric micelles can be used to encapsulate those small-molecule drugs and enhance their prolonged systemic circulation to provide tumor selectivity and decrease side effects. This targeting method is called passive targeting because it relies on carrier characteristics and tumor biology, but it does not contain any specific ligands to bind target tissue or organ.

\subsubsection{Active targeting}

Active targeting can greatly increase the number of delivered drugs to the target than passive targeting [39]. Active targeting is achieved by modifying the surface of polymeric micelles with specific ligands that bind to receptors on the target tumor cells. Active targeting is especially effective in treating cancer due to the overexpression of cancer cell receptors. The attachment of specific ligands to the hydrophilic surface of block polymers can be used to produce active targeting micelles [37]. Such modification will improve the affinity of the polymeric micelles for the target tumor, enhance the drug efficiency and reduce side effects to normal tissues. Another approach of active targeting is to manipulate polymeric micelles' response to specific stimuli unique to disease conditions. Drugs can be released by internal stimuli such as $\mathrm{pH}$, temperature, by external stimuli such as light, heat, or by a combination of both stimuli.

\subsection{Imaging}

Nuclear imaging, magnetic resonance imaging (MRI), and X-ray computed tomography (CT) are used to diagnose cancer and disease evaluation.[38] Contrast agents are introduced to the body to greatly enhances the area of interest in the body. Polymeric micelles have the application of used as delivering contrast agent. For nuclear imaging, contrast agents are made by polymeric micelles loaded with gamma emitters. As a contrast agent of MRI, iron oxide particles are loaded into micelles. CT is less suitable for molecular imaging due to its high concentration of contrast agent requirement. Micelles modified for tumor imaging using $\mathrm{CT}$ have not been reported.

\subsection{Stimulus Responsive Drug Release}

Even though Polymeric micelles are suitable for drug carriers, some problems remain. Polymer micelles are highly associated with premature and insufficient drug release [40]. Ideally, polymer micelles should hold the drug until they are present in the target area, but in reality, they will start to release after accumulating in the tumor interstitium. The way to solve this problem is to increase the interaction between the drug and the hydrophobic core. To achieve adequate drug release, stimuli-responsive PMs are used in tumor-targeted drug delivery

\subsection{1 $\mathrm{pH}$-responsive}

pH-responsive polymeric micelles have become important nanocarriers for drug delivery. Due to increased metabolic rates and aerobic glycolysis, most tumor tissues perform acidic $(\mathrm{pH}$ 6.5-7.2) environments [41]. While blood is relatively basic ( $\mathrm{pH} 7.4$ ), such difference can be exploited to drug release using $\mathrm{pH}$-responsive polymeric micelles. One possible approach to provide $\mathrm{pH}$ sensitivity to polymeric micelles is to incorporate the ionizable groups into polymeric micelles. Once the groups are ionized in acidic conditions, polymeric micelles will decompose and cause the drug to release into tumor tissues.

\subsubsection{Thermo-responsive}

Temperature is one of the most investigated stimuli in drug delivery [40]. The change of temperature on tissue is internal (e.g., inflammation, infection) or external (apply heating device). Since tumor cells can also be damaged by increasing temperature, thermo-responsive polymeric micelle are the most investigated drug-release approach in cancer therapy. Polymers made from thermo-responsive polymeric micelles can undergo a sharp change of physical properties with temperature change, so polymeric micelles will deform and release drugs when the temperature is changed. Those polymers typically have low critical solution temperatures (LCST). The polymer becomes water-soluble below the LCST, becomes insoluble above LCST due to the disruption of hydrogen bonds between water and polymer chains. LCST can be modulated by adding hydrophobic or hydrophilic comonomers to obtain the desired LCST range $\left(37^{\circ} \mathrm{C}-42^{\circ} \mathrm{C}\right)$, which higher than normal body temperature but lower than tumors.

\subsubsection{Ultrasound-responsive}

Ultrasound has been used in therapeutic and diagnostic applications for many years due to its low cost, ease of administration, and deep penetration [42]. Both hyperthermia and non-thermal effects take place when the tissue is exposed with ultrasound. Studies have shown that ultrasound can be used to target micelles and trigger drug release [43].

\section{Conclusion}

In this review, we introduced the morphology, synthesis method of PMs, and their applications in the medical field. From the initial hollow vesicles to the now complex and large smart vesicles, the technology of polymer vesicles is changing with each passing day. In addition to solvent switching technology and Solvent-free technology, more synthesis techniques are developed. Synthetic methods like PISA are likely to become the main way to synthesize polymer vesicles in the future. The double-layer membrane structure of polymer vesicles has good compatibility with biological membranes, and it is an ideal drug carrier in vivo. PMs have good histocompatibility and cell penetration like liposomes when used as drug carriers, but they have higher stability and chemical versatility than liposomes. After the polymer vesicle encapsulates the drug, it can play the role 
of synergism, attenuation, slow-release, and increase drug stability. Its hydrophilic surface shell has a steric stabilizing effect, which can prolong the circulation time in the body and enhance passive targeting Ability: After being attached to the surface of biological molecules, it can also give vesicles active targeting function or other biological effects. However, it should be noticed that the difference between lab experiments and practical applications is still considerably large. The polymer vesicles themselves need to be biodegradable and biocompatible, but their degradation products also need biocompatibility. The degradation products need to be non-toxic and not have harmful side effects on other cells. Moreover, the current PMs are independently developed by various laboratories. They do not have interoperability, which means they only have a single function, resulting in high costs when introducing practical applications. The transportation and storage of polymer vesicles also require further research. Finally, only limited companies can manufacture PMs for practical applications. Therefore, further research needs to emphasize producing PMs with a longer shelf life and better environmental adaptability.

\section{References}

1. P.L. Soo, A. Eisenberg, J. Polym. Sci. B. Polym. Phys. 42, 923 (2004).

2. Q. Liu, S. Chen, J. Chen, J. Du, Macromol. 48, 739 (2015).

3. A. Kishimura, Polym. J. 45, 892(2013).

4. A. Blanazs, J. Madsen, G. Battaglia, A.J. Ryan, S.P. Armes, J. Am. Chem. Soc. 133, 16581 (2011).

5. N.J. Warren, O.O. Mykhaylyk, D. Mahmood, A.J. Ryan, S.P. Armes, J. Am. Chem. Soc. 136, 1023 (2014).

6. N.J. Warren, S.P. Armes, J. Am. Chem. Soc. 136, 10174 (2014).

7. M. Marguet, O. Sandre, S. Lecommandoux, Langmuir 28, 2035 (2012).

8. M. Marguet, L. Edembe, S. Lecommandoux Angew. Chem. Int. Ed. 51, 1173-6 (2012).

9. Y. Moroi Micelles: Springer international ed. New York: Springer 41-50, 2005.

10. M. Ueno, N. Isokawa, K. Fueda, S. Nakahara, H. Teshima, N. Yamamoto, H. Yokoyama, Y. Noritsugu, K. Shibata, K. Miyagawa, S. Tanaka, T. Hirano, A. Fujito, A. Takashima, K. Kanno. World J. Chem. Edu. 32-44 (2016).

11. Vert M. et al. IUPAC Recommen 84, 377 (2012).

12. D.T. Pham, A. Chokamonsirikun, V. Phattaravorakarn, et al. J. Mater. Sci. 56, 2016 (2021).

13. P.C. Heimenz, R. Rajagopalan, Principles of Colloid and Surface Chemistry, Third Edition, (1997).

14. G. Wanka, H. Hoffman, W. Ulbricht, Macromol. 27, 4145 (1994).
15. C. Allen, D. Maysinger, A. Eisenberg, Colloids. Surf. B 16, 3 (1999)

16. D.L. Johnson, M. Polikandritou-Lambros, T.B. Martonen, The Knee 3, 9 (1996)

17. E. Themistou, G. Battaglia, S.P. Armes, Polym. Chem. 5, 1405 (2014).

18. M.T. Popescu, M. Korogiannaki, K. Marikou, C. Tsitsilianis, Polym. 55, 2943 (2014).

19. J. Du, R.K. O’Reilly, Soft Matter 5, 3544 (2009).

20. J. Lee, H. Bermudez, B.M. Discher, M.A. Sheehan, Y.Y. Won, F.S. Bates., D.E. Discher, Biotechnol. Bioeng. 73, 135 (2001).

21. J. Du, S.P. Armes, Langmuir 25, 9564 (2009).

22. J. Du, S.P. Armes, Langmuir 24, 13710 (2008).

23. A.T. Nikova, V.D.Gordon, G. Cristobal, M.R.Talingting, D.C. Bell, C.Evans, M.Joanicot, J.A.Zasadzinski, D.A Weitz, Macromol. 37, (2004).

24. J. Du, Y. Tang, A.L. Lewis, S.P. Armes., J. Am. Chem. Soc. 127, 17982 (2005).

25. J. Chen, Q. Liu, J. Xiao, J. Du. Biomacromol. 16, 1695 (2015).

26. A. Koide, A. Kishimura, K. Osada, W Jang, Y. Yamasaki, K. Kataoka, J. Am. Chem. Soc. 128, 5988 (2006).

27. Y. Anraku, A. Kishimura, M. Oba, Y. Yamasaki, K. Kataoka, J. Am. Chem. Soc. 132, 1631-6 (2010).

28. S. Schrage, R. Sigel, H Schlaad, Macromol. 36, 1417 (2003).

29. S. Ohno, K. Ishihara, S. Yusa, Langmuir, 32, 3945 (2016).

30. R. Takahashi, T. Sato, K. Terao, S. Yusa,. Macromol., 49, 3091 (2016).

31. S. Hauschild, U. Lipprandt, A. Rumplecker, U. Borchert, A. Rank, R. Schu-bert, S. Förster, Small 1, 1177 (2005).

32. H.C. Shum, J.W. Kim, D.A. Weitz, J. Am. Chem. Soc. 130, 9543 (2008).

33. Q. Yi, G.B. Sukhorokov, J. Ma, X. Yang, Z. Gu, Int. J. Polym. Sci. 2015, 756237 (2015).

34. 18H. Gao, O.A. Goriacheva, N.V. Tarakina, G.B. Sukhorukov, ACS Appl. Mater. Interfaces 8, 9651 (2016).

35. S.M. Hartig, R.R. Greene, J. DasGupta, G. Carlesso, M.M. Dikov, A. Prokop, Davidson JM, Pharm. Res. 24, 2353 (2007).

36. C. Gonzato, M. Semsarilar, E.R. Jones, F. Li, G.J.P. Krooshof, WymanP, O.O. Mykhaylyk, R. Tuinier, S.P. Armes, J. Am. Chem. Soc. 136, 11100 (2014).

37. Y. Zhu, Bo Yang, C. Shuai, et al., Prog. In Polym. Sci. 64, (2016).

38. S. Movassaghian, O. M. Merkel, V.P. Torchilin, WIREs Nanomed Nanobiotech. 7, 691 (2015).

39. Q. Zhou, L. Zhang, T. Yang, H. Wu, Int. J.of Nanomedi. 13, 2921 (2018). 
40. C. Oerlemans, W. Bult., M. Bos., G. Storm., J Frank W Nijsen, W.E. Hennink, Pharm. Res. 27, 2569 (2010).

41. J.F. Nijsen, G.C. Krijger. A.D.van het Schip, Anticancer Agents Med. Chem. 7, 271 (2007).

42. G.A Husseini, W.G Pitt, J. of Pharm. Sci. 98, 795811 (2009).

43. M. Kanamala, W. R. Wilson, M. Yang, B.D. Palmer, Z. Wu, Biomater. 85, 152 (2016). 